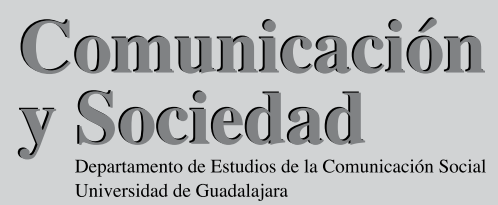

\title{
¿Quién hace la noticia en el Parlamento brasileño? Análisis comparativo de las rutinas de los newsmakers del Senado federal*
}

FRANCISCO SANT'ANNA1

La cobertura periodística del Parlamento brasileño es hecha diariamente por un contingente de más de 200 periodistas. Una parte es contratada por la prensa tradicional, mientras que otra compone las plantillas de los medios de las fuentes del Senado federal (MFSF). El objetivo de este texto es delimitar el perfil socioprofesional, las rutinas productivas, valores y paradigmas periodísticos adoptados por los profesionales de los MFSF, en un estudio comparativo con los de los compañeros de la prensa tradicional, así como la representación social que poseen con relación a los pertenecientes a los MFSF.

PALABRAS CLAVE: medios de las fuentes, territorio profesional, periodista de las fuentes, periodismo legislativo, rutinas periodísticas.
Press coverage of the Brazilian Parliament is done daily by a contingent of more than 200 journalists. One part of that contingent is hired by the traditional press, while another part works for the Federal Senate mass media of source structures (MFSF). The aim of this article is to delimit the social-professional profile, the routines, values and journalistic paradigms adopted by those professionals of the MFSF in a comparative study with their colleagues from the traditional press, as well their social representation they possess in relation to the MFSF.

KEY WORDS: media of the source, professional territory, journalist of the source, parliamentary journalism, journalistic routines.

* Traducción del portugués: Ivan Godoy y Hugo Machin. Revisión y corrección en español: Teresa de Jesús Tovar Peña.

1 Universidad del Legislativo do Brasil (Unilegis) y Núcleo de Estudos sobre Mídia e Política (Nemp) de la Universidad de Brasilia, Brasil.

Correo electrónico: chicosantanna@hotmail.com. 


\section{INTRODUCCIÓN2}

Diariamente dos pelotones de periodistas, cada uno con un poco más de un centenar de profesionales, recorren las instalaciones del Senado federal brasileño (SF) para cubrir los trabajos de los parlamentarios. Un pelotón lo componen reporteros especializados en la cobertura política y parlamentaria contratados por la prensa tradicional, aquí denominados, según el vocabulario profesional, como sectoristas. En el segundo pelotón están los periodistas contratados por los medios del propio SF, aquí denominados medios de las fuentes del Senado federal (MFSF). El Senado federal de Brasil dispone de una emisora de radio que opera en frecuencia modulada y en ondas cortas; un canal de televisión que puede ser captado via satélite, cable y en un canal abierto por el sistema UHF (Ultra High Frequence); un diario impreso, una agencia de noticias y fotoperiodismo, y una radio agencia de noticias. Todos estos medios están igualmente disponibles por Internet. Denominaremos a los profesionales que trabajan para estos medios "periodistas de las fuentes". Los dos pelotones tienen por delante el mismo reto de obtener y difundir informaciones sobre una misma temática y en un mismo espacio físico.

La redacción más grande, perteneciente a la Cámara Alta del Parlamento, es la de Tele Senado, compuesta de 36 profesionales. Radio Senado y la Radio Agencia Senado, juntas, tienen 31 periodistas. La Agencia Senado de Noticias (ASN), 30 y el Diario del Senado, 18.

¿Quién es el profesional que actúa en la producción de noticias difundidas por estos medios de las fuentes?3 ¿Qué valores periodísti-

2 La investigación relatada en este artículo se incluyó en la tesis de doctorado Média de source, un nouvel acteur sur la scène journalistique brésilienne, presentada, en octubre del 2007, por el autor en la Universidad Rennes 1, en Francia. La tesis está disponible, en francés, en http://www.crape.univ-rennes1.fr/documents/theses/theseSant\%27Anna.pdf. Este texto fue seleccionado para ser presentado en el II Seminário Internacional Media, Jornalismo e Democracia, "Jornalismo e Actos de Democracia", organizado por el Centro de Investigação Media \& Jornalismo y la Escola Superior de Comunicação Social-Instituto Politécnico de Lisboa, el 13 y 14 de noviembre del 2006.

3 El concepto "medios de las fuentes", creado por el autor del texto, busca 
cos orientan sus rutinas profesionales? En la búsqueda de respuestas, realizamos, en el 2005, una investigación de campo. Nos valimos de consulta directa a los profesionales con la aplicación de cuestionarios self-answering. Los sectoristas fueron igualmente investigados para servir como un grupo de control. El objetivo era recoger informaciones capaces de trazar la "calificación, preferencias, rutinas y valores profesionales y relaciones" de los entrevistados “con la profesión”. La investigación buscó evidenciar si periodistas contratados por estructuras que operan el llamado "periodismo institucional" poseen perfil y rutinas diferentes de los compañeros de la prensa tradicional.

La encuesta se respaldó en la misma metodología utilizada con periodistas norteamericanos por Weaver y Wilhoit's, en 1982 y 1992, cuyos resultados fueron publicados en 1986 y 1996 (Weaver et al., 1986 y 1996), respectivamente. Investigación semejante fue realizada, en la década de 1980, por McMane (1992) para comparar a los periodistas franceses con sus compañeros británicos, alemanes y estadounidenses. ${ }^{4}$ La utilización de esta metodología en gran escala y en diferentes países aporta seguridad al estudio del territorio informativo que constituyen los medios de las fuentes y de los profesionales que para ellos trabajan.

Con la investigación fue posible trazar el perfil social, las influencias y preferencias culturales, el patrón de vocación profesional, valores deontológicos, rutinas, dificultades y presiones en la producción de informaciones. Se identificaron los principales interlocutores de los profesionales, así como su nivel de satisfacción con el trabajo. Se pudo trazar, además, la representación social que tienen en relación con el periodismo de manera general y, de forma más específica, con el trabajo que desarrollan. Los datos contribuyen a reflexionar sobre el papel que tales profesionales juzgan

identificar medios de comunicación de masa que son mantenidos por las propias fuentes. Para mejor comprensión del concepto ver Sant'Anna, F. (2005). Mídia das Fontes -o difusor do jornalismo corporativo, Brasília: Casa das Musas, o el artículo sobre el mismo tema disponible en: http:// www.bocc.ubi.pt/pag/santanna-francisco-midia-fontes.pdf.

4 Trabajo semejante también se realizó en otras naciones, como, por ejemplo, Heloiza Herscovitz (2000) y Joaquim Negreiros (2004) con periodistas brasileños y portugueses, respectivamente. 
estar ejerciendo en el seno de la sociedad y contestar una de las preguntas de la investigación en cuanto al surgimiento de un nuevo periodismo, uno practicado por las propias fuentes y que por eso podría clasificarse como un "periodismo de influencia", entendido como uno que busca el convencimiento social. Todos estos elementos fueron objeto de comparación con el grupo de control formado por los sectoristas.

\section{PERFIL SOCIAL}

El sistema sociocultural en el que está insertado el periodista es un elemento importante en el proceso de produccción de las noticias. "La construcción de la noticia sufre una acción conformadora por parte del sistema sociocultural en el cual se inserta" (Sousa, 2000: 85). El perfil sociocultural de los periodistas de los MFSF es bastante semejante al de los sectoristas; en tesis, está sujeto a la misma "acción conformadora" que enfrenta su compañero de prensa tradicional. En promedio, tal perfil tiene poco más de 39 años de edad y está desde hace más de 17 años en la profesión. Es un perfil ligeramente más joven, tanto en edad como profesionalmente, que el del grupo de control. Actúan en el SF hace 5 años y 2 meses, en promedio.

Una diferencia entre los dos grupos es la forma de selección, reclutamiento y el régimen de contrato de trabajo. Todos los periodistas de las MFSF fueron contratados mediante cuatro concursos públicos de alcance nacional. El periodista aprobado adquiere un estatus de funcionario de carrera (Régimen Jurídico Único) que le garantiza estabilidad en el trabajo y un plan de carrera. Condiciones raras en el sector privado. Dimisión, solamente en consecuencia de faltas graves e investigadas en un largo proceso administrativo. Esta condición, al parecer, proporciona una independencia profesional ante eventuales abusos editoriales o presiones de los niveles jerárquicos superiores o por parte de parlamentarios. Es una condición importante para que se trabaje en una institución política.

Los periodistas estables demuestran un mayor compromiso con los objetivos centrales del medio, interesados en reforzar la transparencia de la actividad legislativa y no los proyectos personales de los senadores (Freitas, 2004: 26). 
Con relación a las fuentes, en especial con los senadores, el periodista no está técnicamente subordinado al parlamentario.

En el periodismo tradicional, la trayectoria profesional se caracteriza por el deterioro de las relaciones de trabajo y la sumisión a los intereses empresariales (Rieffel, 2001 y Ruellan, 2001). En el mercado, pesa significativamente lo que en el lenguaje popular se llama capacidad de "vestir la camisa de la empresa".

El periodista del MFSF, como su semejante, es un profesional que ideológicamente se autocalifica de "izquierda" (39\% se identifican así y $41 \%$ de centro-izquierda). En una escala de 1 a 10, donde 1 es "extrema izquierda" y 10 equivale a la "extrema-derecha"; con un promedio de 3.37, ellos se muestran un poco menos a la izquierda que sus semejantes, 3.05. Los reflejos del patrón ideológico son diferentes cuando se analiza el grado de vínculos sindicales y partidarios. La cantidad de sindicalizados es mucho más grande entre los primeros, $68 \%$, contra $58 \%$. Las exigencias patronales del sector privado tal vez estén en el origen de esta realidad. En cuanto a vinculaciones partidarias, $8 \%$ de los profesionales del SF están afiliados a partidos políticos, contra $2 \%$ entre los sectoristas.

El profesional típico, objeto de nuestro estudio, se reveló como hombre (55\%), blanco (82\%), casado (58\%), y en términos de religión, cuando no es ateo (31\%), es cristiano. Los cristianos se dividen básicamente entre el catolicismo (37\%) y el espiritismo (15\%). Llama la atención el hecho de no haber sido identificado en la muestra del grupo de test ningún periodista negro. En la prensa tradicional, los negros representan $10 \%$. Con relación a los mestizos, las proporciones son semejantes: $17 \%$ y $16 \%$, respectivamente. La concentración más grande de periodistas blancos en el SF puede ser una consecuencia del modelo de selección de los contratados. El ejercicio del periodismo, en Brasil, exige estudios universitarios de cuatro años. La enseñanza universitaria todavía es muy elitizada y las minorías étnicas no tienen un acceso tan amplio a esos pupitres. ${ }^{5}$ En la selección, el SF privilegia además los que

5 Varias universidades públicas pasaron a aplicar en este nuevo milenio cuotas raciales en una política de discriminación positiva, para reducir las diferencias de nivel educativo entre los grupos étnicos. El Congreso Nacional examina un proyecto de ley que hace universal tal medida. 
poseen maestría y doctorado. En tesis, quien tiene más años de estudios tiene más chance de ser seleccionado. Casi la mitad de la plantilla del Senado (47\%) posee un diploma de posgrado: máster6 (23\%), maestría $(20 \%)$ y doctorado $(4 \%)$.

\section{INFLUENCIAS Y PREFERENCIAS CULTURALES}

Por interferir en la actividad periodística, buscamos identificar los elementos culturales con relación al consumo de literatura, de producción cinematográfica, etcétera, así como las aspiraciones personales que actúan sobre los periodistas investigados. Se reveló que el "periodista de la fuente" aprecia a los autores brasileños, lo que revela, eventualmente, la valorización de lo que es nacional, al contrario de lo que es importado. La lectura predilecta es esencialmente de ocio, en especial novelas (35\%), historias de aventura y ficción (14\%) y temas históricos y épicos (14\%). Los libros técnico científicos y de periodismo no se destacaron. En cuanto al cine, uno de cada cuatro profesionales no tiene preferencia específica. Entre los demás, los dramas $(26 \%)$, documentales $(10 \%)$ y las comedias $(10 \%)$ son los predilectos. La tercera parte tiene una preferencia clara por el cine europeo (España, Italia y Francia, principalmente), 14\% por el cine nacional y $18 \%$ por el norteamericano. ${ }^{7}$

Es bajo el interés por la prensa internacional. Prácticamente, seis de cada diez profesionales del SF no tienen el hábito de leer periódicos

6 En la categoría máster agrupamos los cursos de posgrado lato sensu, dirigidos principalmente al aspecto de capacitación y no a la investigación académica.

7 Aquí también hay semejanza con los sectoristas, que prefieren autores nacionales, el 44\%. Los libros históricos y épicos representan $26 \%$; novelas, el $14 \%$, y la ficción/aventura, $12 \%$. Dos de cada diez sectoristas no tienen una predilección específica por películas. Entre los demás, el drama (14\%), los filmes de arte o de autor (14\%) y las obras de ficción (12\%) dominan las preferencias. Igualmente, $30 \%$ no tienen debilidad por ningún país; 38\% optan por el cine europeo. El cine brasileño es el predilecto de solamente $8 \%$ de los periodistas, mientras que a 14\% le atrae el de Estados Unidos. 
de otros países -entre los sectoristas, la situación es al revés, 56\% leen habitualmente. La parte restante (41\%) le da prioridad a las noticias de "política" e "internacional" difundidas por los periódicos y revistas norteamericanos. La prensa de Argentina, principal socio brasileño en el MERCOSUR, ya surge como segunda fuente de lectura periodística para los dos grupos investigados. El interés por las noticias argentinas tiene la misma intensidad que el manifestado en cuanto a las inglesas y es superior a las francesas (Le Monde, principalmente). El medio preferido es Clarín. El Página 12, periódico considerado más de la izquierda, y teóricamente, más cercano al perfil ideológico de los periodistas, no fue mencionado por ninguno de los investigados.

A siete de cada diez "periodistas de las fuentes" -y seis de la prensa tradicional- les gustaría hacer un curso de posgrado en Europa, que se muestra como la región preferida, $62 \%$, para un eventual trabajo en el extranjero. La preferencia por Europa, al contrario de Estados Unidos, difiere de lo constatado por Heloiza Herscovitz (2000) con periodistas brasileños que actúan en el estado de São Paulo. Eso puede ser un reflejo de los hechos ocurridos en Nueva York el 11 de septiembre del 2001 y la política internacional norteamericana adoptada desde entonces.

\section{VOCACIÓN PROFESIONAL}

Nuestro "periodista de las fuentes" optó por esa profesión debido, principalmente, a razones de "vocación personal". ${ }^{8} \mathrm{La}$ atracción de $51 \%$ del grupo por la actividad de redacción, en sí misma, puede interpretarse como una preferencia por la dimensión del periodismo como una actividad expositiva. Una manera de ver el periodismo, esencialmente, como "la descripción de los hechos" (Negreiros, 2004: 90). Razones relacionadas a la "fascinación y visibilidad profesional" abarcan una cantidad cercana a la cuarta parte $(23 \%)$ de la plantilla de periodistas del SF. Un $21 \%$ escogieron motivos de compromiso ideológico, como

8 La encuesta organizó en cinco grupos temáticos las razones que llevaron a ingresar en el periodismo: fascinación y visibilidad profesional; compromiso ideológico; facilidades y beneficios materiales; influencias personales externas; y perfil vocacional personal. 
el deseo de informar a las personas y transformar a la sociedad.9 Para éstos, la profesión aparenta "dar un permiso especial a sus miembros, como un mandato de tipo moral o intelectual o incluso legal" (Travancas, 1983:108).

Normalmente, el periodista es un individuo visto por la sociedad como una persona que circula por las esferas del poder político y financiero y a la cual le gusta estar cerca del poder (ibidem: 96-97). La condición de cercanía al poder sería, incluso, la principal atracción de la profesión (Ribeiro, 1994: 205). Sin embargo, los dos grupos investigados ignoraron el deseo de estar cercanos al poder como una de las razones de ingreso en la profesión. La misma posición mantuvieron en cuanto a las razones más pragmáticas y materiales, como sueldo o ascención social.

Las percepciones de los dos grupos sobre el papel de la prensa en la sociedad son semejantes. ${ }^{10}$ Los datos revelan, sin embargo, que los periodistas del SF le dan una mayor importancia al papel social de la prensa y reconocen en ella una capacidad de intervención en la opinión pública más grande que lo que creen los sectoristas. También creen que es de significativa importancia el papel que ejercen los MFSF -lo que no es reconocido en la misma intensidad por los demás. Mientras los primeros consideran de "importancia significativa" (7.04 puntos) la información difundida por el sistema de comunicación del Senado para la sociedad, el segundo grupo le atribuye una "importancia mediana" (5.37 puntos).

9 La vocación personal (60\%) y el compromiso ideológico (32\%) son las principales motivaciones de los sectoristas. El aspecto de la fascinación y visibilidad profesional se refiere a solamente $6 \%$ de esos profesionales.

10 Para la evaluación de este aspecto se aplicó una escala de siete niveles, "A" a "G", donde "A" equivale a cero (sin ninguna importancia) y "G" equivale a diez (extremadamente importante). Esta modalidad de abordaje se elaboró a partir del principio de la Escala de Likert, que consiste en una metodología del campo de la psicología para medir las variables relacionadas con las actitudes de los individuos. Es un instrumento que permite identificar la aceptación o rechazo de un ser humano respecto a un fenómeno ocurrido en el ámbito de la naturaleza o de la sociedad (Pasquali, 1996: 432). 


\section{VALORES DEONTOLÓGICOS Y RUTINAS PROFESIONALES}

Los periodistas de los MFSF demuestran tener valores ético-profesionales más rígidos que sus compañeros de la prensa tradicional. En una escala de rigidez ética de tres puntos $(1=$ flexible, $2=$ regular, $3=$ rígida) los "periodistas de las fuentes" obtuvieron un promedio de 2.70, mientras el grupo de control registró 2.43. Para alcanzar este resultado, se presentaron marcos éticos asociados a ocho situaciones comunes al cotidiano periodístico. De forma general, no están abiertos a todo método o estrategia para obtener una información. El estímulo a que ocurra un hecho socialmente condenable solamente para transformarlo en noticia es la práctica más rechazada, 2.98, por parte de los periodistas de las fuentes. ${ }^{11} \mathrm{El}$ rechazo es más intenso que el comprobado en cuanto a uno de los dogmas de la profesión, que es el secreto sobre la fuente (2.88). Uno de cada diez periodistas admite, en ciertas condiciones, no respetar el off the records. ${ }^{12}$

En una sola situación los dos grupos presentan un patrón ético de igual intensidad. El pago de fuentes para obtener informaciones es rechazado a razón de 2.8 puntos. La disimulación de la identidad profesional, en la proporción de 2.49 y la posibilidad de obtener empleo en una empresa $u$ oficina del gobierno para clandestinamente conseguir informaciones (2.75) son métodos condenados en el ámbito del SF en una intensidad de $12 \%$ superior al comprobado entre los sectoristas. ${ }^{13}$ La prensa tradicional es más abierta a la utilización sin autorización de documentos o fotos de personas físicas o de instituciones gubernamentales y privadas. Los profesionales del SF actúan preferencialmente con documentos e imágenes autorizados para la divulgación.

11 En el grupo de control, este tópico obtuvo 2.88 puntos.

12 En la prensa tradicional, 2.58 puntos, muy cercano a la opción que "admite en determinadas situaciones el rompimiento de un acuerdo y revelar la fuente de información”. Entre los profesionales escuchados, 22\% admiten esta posibilidad.

13 Los resultados de los sectoristas fueron: "disimular la identidad profesional (2.49); obtener un empleo en una empresa, o en una oficina del gobierno para conseguir informaciones clandestinamente" (2.47). 


\section{VALORES DEONTOLÓGICOS}

La utilización indebida de documentos personales y fotos íntimas, muy apreciada por la prensa del tipo people, provoca un rechazo más grande, 2.85 puntos. Según Thompson (2005: 81), una vez aceptada la idea de que se pueden retirar las cortinas que cubren la cúpula del poder, se vuelve difícil mantener una distinción estricta entre los secretos referentes al ejercicio del poder y los secretos de la vida privada. Temas que reciben prioridad por los criterios de noticia de algunos medios privados, como la vida personal de los políticos, no son blanco del interés de los MFSF, a no ser que intervengan en el proceso político parlamentario. El uso sin autorización previa de documentos confidenciales, públicos o privados, es admitido solamente por la tercera parte de los periodistas de las fuentes. Los sectoristas se mostraron más bien flexibles. Este procedimiento es más factible entre ellos en lo que se refiere a la cobertura del Parlamento brasileño.

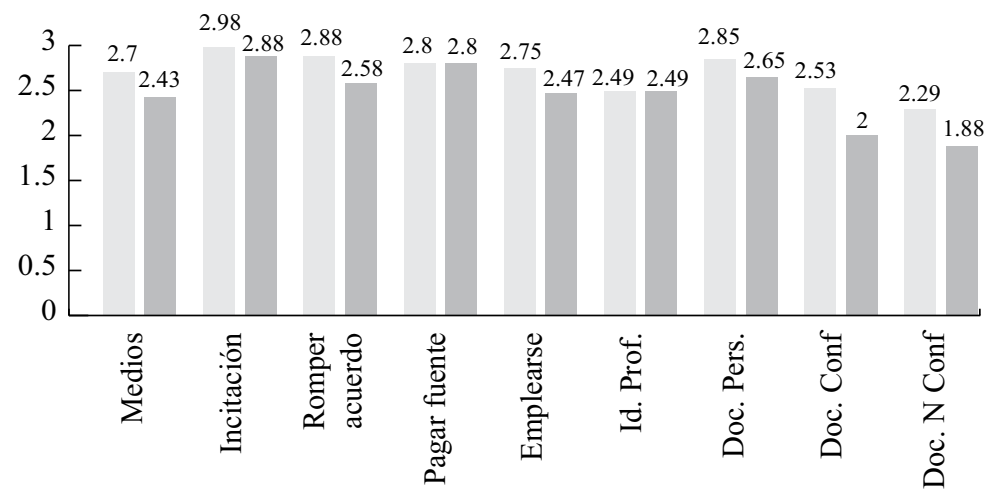

Senado

Sectorista

La diferenciación de valores entre los dos grupos de periodistas puede ser una consecuencia directa de los patrones editoriales a que están sometidos. La prensa tradicional sufre interferencia de inspiración mercadotécnica en la búsqueda de la "noticia exclusiva", de audiencias más grandes y, por eso, no siempre adopta límites socialmente aceptables. Un punto de constante polémica, por ejemplo, es el uso de cámaras o 
micrófonos ocultos. Un patrón ético menos rígido puede atribuirse, de la misma manera, al modelo de periodismo más investigativo y especulativo practicado por el sector. Los medios del Senado, por su parte, tienden a trabajar con los hechos visibles, evitando investigaciones y especulaciones. Los MFSF tienden a poner en una posición de menor importancia periodística hechos ajenos al blanco noticioso principal, el Parlamento.

\section{PARÁMETROS PERSONALES VERSUS PATRONALES}

En su actividad cotidiana, todo periodista necesita administrar el conflicto entre sus valores personales y los criterios editoriales patronales. La información que se difunde es elaborada a partir de una interacción de fuerzas situadas a nivel personal, social, así como del medio físico y tecnológico y, claro, del patrón. Es una tarea ardua y riesgosa, pues lo que estará en juego, además de la fidedignidad periodística, es la credibilidad profesional, la estabilidad en el empleo y el mismo futuro de la carrera del periodista. En los medios del SF, la percepción sobre los límites editoriales implantados por la Casa no son linealmente claros en la mente de sus profesionales. Y existe de hecho un conflicto entre los valores editoriales personales y los reglamentados por la institución.

Los periodistas de las fuentes afirman que los dos principales parámetros editoriales establecidos por el Senado son "rapidez" (37\%) y "precisión" (33\%). Los datos revelan que incluso en el periodismo considerado institucional, la competencia entre los medios impregnó el "factor tiempo" en la cultura periodística y en los valores del newsmaking, suplantando el carácter "noticia" de los hechos (Schlesinger, 1983).

La obsesión por la velocidad en la producción y distribución de noticias alteró los criterios de noticia en la producción periodística contemporánea. El valor de la noticia se encuentra más en su instantaneidad que en su credibilidad (Adghirni, 2004: 1).

La velocidad de la divulgación provoca un choque conceptual. En la cultura periodística "divulgación rápida" siempre fue enemiga de la "precisión". Incluso en la cultura popular los dos aspectos no combinan. 
El dictado "la prisa es enemiga de la perfección" retrata el imaginario social brasileño. Profesionales y medios trabajan siempre en un frente bastante arriesgado, donde la competencia y credibilidad son constantemente puestas a prueba. Los periodistas de los dos grupos se sienten perjudicados debido a esa exigencia editorial. Sin embargo, en los manuales de redacción de los MFSF, no aparece el aspecto rapidez entre las exigencias editoriales. Este patrón puede justificarse por el hecho de que el SF posee dos agencias de noticias en "tiempo real" (una de texto y otra de informaciones de radio) y de que difunde sus noticias en la radio y la televisión, medios que tienen como perfil la instantaneidad de la noticia. La precisión, sin embargo, es insistentemente mencionada. Radio Sena$d o$, además de ser categórica con relación al aspecto precisión -"no puede haber dudas sobre la información que va al aire"-14 refuerza la necesidad de que los reportes sean producidos a partir de múltiples fuentes.

El uso de varias fuentes y de citas entre comillas es una técnica que protege al periodista de cometer imprecisiones y se muestra como una alternativa a la exposición de su propia opinión sobre los hechos tratados (Tuchman, 1972: 660). Así, puede expresar un análisis con el cual esté de acuerdo, sin que tenga que firmar abajo como de su autoría. Evita la exposición a las presiones y cobros, internos y externos. Es lo que Charron (2002: 1) denomina hablar de lo que se piensa incitando a los otros a expresarse -"Parler de soi en faisant parler les autres"-. La jerarquía que le da a la opinión de las fuentes contribuye a transmitir un punto de vista, que sería equivalente a la opinión que el "periodista de la fuente" emitiría en caso de que fuera autorizado a hacerlo.

Sin embargo, en el imaginario de los "periodistas de los MFSF", escuchar los diversos lados de la historia no parece estar en las "prioridades patronales". Otros aspectos, como "objetividad/imparcialidad" (27\%) y "análisis e interpretación de los temas para el público" (25\%), forman un segundo nivel de parámetros editoriales, los cuales los periodistas creen estar obligados a seguir.

Entre las "referenciales personales" de los periodistas de las fuentes, el análisis e interpretación de los temas para el público, con $47 \%$ de los

14 Cfr. Manual de redacción de Radio Senado. Disponible en: http://www.senado.gov.br/radio/senadoFM.asp 
mencionados, objetividad/imparcialidad (37\%) y transparencia (31\%) son los que se destacan. El resultado evidencia un conflicto entre los valores personales y las normas superiores que se cree tener que obedecer, una clara inversión de prioridades entre patrones y empleados. El aspecto "divulgación rápida", considerado la principal exigencia del patrón, aparece en la cuarta posición de prioridad personal, al lado de "precisión" (27\%).

Los valores personales existentes entre "periodistas de las fuentes" y los de la prensa tradicional tienden a ser semejantes. ${ }^{15}$ El parámetro periodístico personal más importante es el "análisis e interpretación de los temas para el público", 54\%. Esto demuestra la preservación de lo que Bourdieu (1980: 91) denominó habitus -en este caso un "habitus periodístico"- entre los profesionales de los MFSF. Este habitus, según el autor, resulta del condicionamiento a prácticas individuales y colectivas, que interfieren históricamente en las formas de pensar y de actuar. Es una referencia más intensa que eventuales reglas y normas internas formales, y permite articular conjuntamente las prácticas y las representaciones profesionales. La diferencia entre los dos grupos es que el primero, del SF, no se siente autorizado a practicarlo, mientras que el segundo cree ser ésta la preferencia de sus patrones.

La principal divergencia entre los dos grupos profesionales se encuentra en la referencia "transparencia de los hechos públicos", que es importante para los profesionales del SF; se convierte casi en un patrón de dogma corporativo, pero solamente obtuvo $10 \%$ de las menciones de los compañeros sectoristas. Se encuentra expresada en los estatutos de la Asociación de los Profesionales de Comunicación del Senado Fede-

15 Entre los sectoristas, el aspecto "análisis e interpretación de los temas para el público", fue el más citado tanto cuando se refería a los límites empresariales, $60 \%$, como cuando era personal, $54 \%$. La necesidad de contar con una "pluralidad de las fuentes" es la segunda referencia de los sectoristas, sea desde el punto de vista editorial de las empresas, 36\%, sea en la visión personal de aquellos, $40 \%$. En un tercer plano se constata un conflicto de valores entre lo que los periodistas desean, precisión $-32 \%$, y aquello que sus patrones exigen- divulgación rápida, $28 \%$. Objetividad e imparcialidad aparecen enseguida como cuarto parámetro personal, $28 \%$, y empresarial $24 \%$. 
ral (COMSEFE) -fundada para defender internamente los intereses del grupo profesional. El texto expresa que es un deber:

Defender y promover la transparencia de los hechos públicos y el acceso democrático y universal a las informaciones de interés de la sociedad (COMSEFE, 2003).

Se debe destacar que la cuestión de "transparencia", como referencia editorial, está en el origen de la creación de toda esta estructura informativa: "Había la intención de garantizar transparencia a la actuación del Senado y de promover la democratización de la información", (Senado, 2003: 7). El ciudadano tiene derecho al acceso a la totalidad de los trabajos legislativos, "con exención y transparencia", destaca el Manual de la Tele Senado (1999). "Nuestra tarea-complementa el mismo- es mostrar el Legislativo como institución viva, con diferentes facciones políticas y diferentes corrientes de opinión sobre todos los temas". Sin embargo, transcurridos poco más de una década desde la creación de los MFSF, en la percepción de los profesionales este parámetro no parece ser prioridad máxima en la política editorial definida por el Senado.

En resumen, podemos decir que la noticia perfecta, para el periodista del Senado, es la que interpreta y analiza los hechos públicos con imparcialidad y propicia transparencia. El periodista se ve, sin embargo, obligado a producir una información de "rápida divulgación, precisa, imparcial y objetiva". El que le hace competencia directa está personal y empresarialmente orientado a producir una información que "analice" igualmente los hechos públicos y que sea "plural y precisa".

Este patrón editorial repercute incluso en la estructura organizativa de las funciones internas de cada sector. En el Senado, los profesionales son divididos básicamente entre reporteros y editores. 16 En la

16 En la categoría reportero incluimos la función de "productor", pues tiene características de procuración y obtención de datos, semejantes a las de un reportero. Editor es el profesional con poder de reformular textos, de cortar, alterar, dar mayor o menor visibilidad a una noticia, sea en la radio, en la televisión o en la prensa escrita. 
prensa tradicional, además de esas dos funciones, existe la función de articulista o columnista, una mezcla de reportero y redactor que posee autonomía para producir textos cargados de fuerte contenido analítico, opinión personal, además de un espacio fijo y periódico, denominado columna, para divulgarlos.

Las funciones de articulista y comentarista no existen en los MFSF. El acto legal que disciplina los objetivos, la utilización y el funcionamiento de los medios de comunicación del SF determina a los procesos internos de producción y difusión de noticias "con carácter apartidario, imparcial y no opinativo" (Senado, 2002). Cabe a los entrevistados el análisis o comentario de los hechos. La exigencia editorial es bien internalizada en el grupo profesional y agrada a una parcela significativa de los periodistas de las fuentes. Ocho de cada diez periodistas reconocen que actuar en los medios del Senado significa producir textos neutrales, sin juicio de valor, análisis o interpretación. Ellos creen que, con la política editorial del SF, cabe al público juzgar los hechos por sí mismo.

Cuando se les preguntó si los valores periodísticos patronales coinciden con los personales, $45 \%$ dijeron que sí y que los adoptarían voluntariamente en sus rutinas. Los datos reflejan una realidad nacional donde el periodista asalariado perdió autonomía profesional, dejó de emitir su opinión y su función social se debilitó ante la opinión corporativa de los medios de comunicación (Ribeiro, 1994: 22). Es fundamentalmente un "soldado de infantería" (Mathien, 1992: 148), cumpliendo órdenes según la pauta del día. Prevalece el modelo del "obediente", del "cumplidor de tareas", del tâcheron, que cumple las estrategias superiores, una pieza del engranaje montada según las estrategias económicopolíticas de los generales del sector.

Sin embargo, entre los profesionales de los medios del Senado conviven dos concepciones diferentes de hacer periodismo. Prácticamente, otra mitad de los periodistas (49\%) afirma que si pudiera valerse libremente de sus valores personales, escribirían textos críticos y de opinión. Para ellos es un deber del periodista interpretar para la opinión pública los riesgos de las decisiones políticas. ${ }^{17}$

17 De la misma manera piensan mayoritariamente los integrantes del grupo de control: $88 \%$ adoptarían este comportamiento a partir de su modo personal 


\section{RUTINAS INFORMATIVAS}

Los periodistas de las fuentes están sometidos a un proceso de construcción de la noticia más jerarquizado y con el poder de decisión más centralizado que sus congéneres. Fueron identificados dos tipos de jerarquización. Para una mayoría de pequeña magnitud, 53\%, la relación de los temas que deberán ser seguidos es diariamente definida en instancias periodísticas superiores y entregada arbitrariamente a los periodistas. En este grupo se concentra la mayor parte de los reporteros, redactores y productores vinculados a los noticieros (noticieros de televisión, de radio, agencia de noticias y diario impreso). El segundo grupo reúne de forma mayoritaria profesionales que participan en programas especiales, de entrevistas, debates de estudio y en la producción de documentales. En este caso normalmente existe una inversión: la selección de los temas es hecha por el profesional que está en la punta, pero es sometida a la aprobación superior.

Normalmente, la confección de la pauta, sea diaria o semanal, ocurre durante reuniones coordinadas por el jefe de redacción, en las cuales participan los jefes de reportaje de cada turno y los editores jefes de los productos periodísticos. Sugerencias de reporteros son analizadas, pero difícilmente participan en el proceso deliberativo. La pauta considera los trabajos previstos para las diversas instancias internas del Parlamento, tales como análisis y votación de proyectos, audiencias públicas, trabajo de las comisiones permanentes y temporales, orden del día del pleno, entre otros.

En el criterio de distribución de los periodistas, el espacio físico institucional prevalece sobre la referencia temática. Así, un reportero que cubre la Comisión de Asuntos Sociales (CAS), por ejemplo, redactará sobre todos los temas ahí tratados. En un mismo día la CAS podrá analizar asuntos tan diferentes como salud, educación, cultura, derechos sociales, minorías, televisión, radiodifusión, etcétera. Una propuesta

de hacer periodismo. En el grupo de control, el conflicto entre concepciones personales y patronales aparenta ser menor, pues $74 \%$ de los periodistas informaron que sus superiores esperan un texto crítico y analítico capaz de alertar a la opinión pública. 
aprobada en la CAS seguirá para el análisis de otra comisión o votación final en el pleno del Senado, pero el reportero de los MFSF no seguirá con la misma pauta. El tema será heredado por otro profesional, aquel que esté designado para cubrir el espacio físico institucional siguiente.

Como en la prensa tradicional, los MFSF también sufren una acción externa de sensibilización de sus gatekeepers. Están sometidos a un mismo patrón de interferencia, a las mismas técnicas de "contra agendación" ejercidas por las fuentes. La prioridad editorial es el trabajo legislativo institucional, pero las asesorías de prensa de los parlamentarios envían sugerencias de pautas referentes a la acción de cada uno de ellos. Entidades externas al Parlamento también buscan influenciar en el contenido a ser difundido. Normalmente son sugerencias sobre temas que son analizados por el SF. Por ejemplo, en el 2005, ONG's y entidades ambientales buscaron pautar sus puntos de vista referentes al Código Forestal Brasileño. El mismo procedimiento realizaron empresas e instituciones ligadas a las cuestiones de aviación civil, explotación y distribución de gas, micro y pequeñas empresas, etcétera. Estos temas estaban siendo regulados por nuevas propuestas de leyes.

En la Tele Senado, las pautas aprobadas en las reuniones son consolidadas en un documento denominado Diario de la Pauta $(D P)$. Versiones impresas son ofrecidas en la redacción y una copia electrónica es enviada a todos los profesionales. El $D P$ cuenta con una selección preliminar de informaciones sobre cada tema seleccionado, datos complementarios (estadísticas, biografías, opiniones de grupos de interés, etapa de trámite de proyectos de ley, eventuales consecuencias, etcétera), así como posibles fuentes a ser escuchadas, sus teléfonos, locales de trabajo, etcétera. Todo es previamente elaborado por un equipo denominado "Producción", formado por periodistas que actúan la mayor parte del tiempo dentro de la redacción, estableciendo contactos telefónicos, agendando entrevistas, siguiendo los noticieros e informaciones en Internet, recogiendo datos preliminares con todas las fuentes posibles (documentales y humanas).

El $D P$ pretende optimizar el trabajo del reportero de campo, permitiendo más rapidez en el desarrollo de su tarea diaria que, en muchos casos, representa más de una nota en el mismo día. Además del tema, el documento define también el profesional que lo realizará. En la televi- 
sión, cuando los reporteros llegan a la redacción, al inicio de sus turnos de trabajo tienen la obligación de conocer su tarea del día leyendo el $D P$. Les toca complementar la investigación preliminar e ir hasta el local de cobertura con el equipo técnico -camarógrafo y ayudantes. El DP delimita también la forma como los jefes de reportaje de cada turno administran sus equipos de trabajo. El margen de maniobra de estos profesionales es restringida, pues las prioridades editoriales ya fueron previamente definidas para atender tanto a las expectativas de los editores de los productos periodísticos sobre las prioridades político editoriales de las instancias jerárquicas superiores. Con el reportero en campo, el desarrollo de su trabajo es seguido por el editor del producto periodístico al cual se destina. Tiempo y tamaño de la nota, resultados, necesidad del uso de infografía, imágenes de archivo, abordaje, texto, todo es blanco de un permanente entendimiento entre el reportero, que está en campo, y su editor, que permanece en el interior de la redacción. Un entendimiento previsto y regulado por las normas internas.

La función clave de los jefes de reportaje de cada turno tiene una naturaleza burocrática y busca concretar las metas planificadas por la jerarquía editorial. La opinión personal de este profesional poco influye en la orientación de los reporteros y en el contenido de sus trabajos. Esta es una tarea de los editores. Si en la BBC inglesa el editor jefe de guardia es considerado the God of the day (Schlesinger, 1987: 144), podríamos decir que en la Tele Senado el poder está en el Diario de la Pauta, es la Biblia del día.

\section{AUTONOMÍA E INTERFERENCIAS \\ EN EL PROCESO PERIODÍSTICO}

Esta rutina periodística, por la cual el reportero es una especie de "soldado de infantería" (Mathien, 1992: 148), obedeciendo a las órdenes y a la planificación de sus comandantes, es común en la prensa brasileña. Aunque el reportero/soldado tome la iniciativa, sin autorización previa, de cubrir un tema, la divulgación de su trabajo, importancia y realce, estarán condicionados a la decisión superior.

Aunque sea una realidad común a diversas estructuras periodísticas, la realidad de los MFSF revela, sin embargo, una concentración del 
poder deliberativo más expresiva que la verificada entre los sectoristas que cubren el Parlamento. Para efecto de comparación, el porcentaje de sectoristas que reciben la pauta previamente definida sin su participación es cuatro veces menor que en los MFSF. En la prensa tradicional, $56 \%$ de los periodistas afirman que no poseen la libertad de cubrir los temas que desean. En el SF el cuadro es más agudo, 71\%. En ambos medios, la libertad del profesional de información tiene como límite el proyecto de la empresa (ibidem: 270). La autonomía periodística de los periodistas de las fuentes se amplía cuando se refiere a la definición del contenido, del ángulo ${ }^{18}$ de abordaje de los reportajes $-51 \%$ afirman tener libertad de decisión-, así como en la definición de fuentes que serían escuchadas, 53\%. Entre los sectoristas los porcentajes son, respectivamente, de $58 \%$ y $80 \%$.

En el día a día, el periodista del SF se vale de un proceso de retroalimentación bastante común a la actividad profesional, descrito por el sociólogo francés Pierre Bourdieu (1997: 9) como "efecto circular circulante". Una especie de efecto dominó donde el contenido de un medio influencia el de otro. La visión de los hechos de un profesional interfiere en la del compañero, aunque estén en medios competidores. Esta rutina de "copia intermediaria" (ibidem: 166) es un instrumento de complementación, profundización e incluso de rectificación que algunos medios, en especial los estructuralmente más frágiles, son obligados a emplear.

La investigación constató que esta retroalimentación ocurre en los dos grupos analizados, y en el SF se da en dos niveles. Uno interno, donde un medio del Parlamento influencia la producción de otro (en

18 Aquí nos valemos de la noción de "ángulo", detallada por Ruellan (1993: 153-154) que cubre diferentes aspectos, en especial la noción de importancia cuantitativa, profundidad, estilo de redacción o punto de observación. La definición del ángulo puede influenciar en la forma de desarrollar el trabajo, en la manera de relatar (por ejemplo, el uso de un personaje para simbolizar una realidad colectiva) de escribir (privilegiando el punto de vista de una o varias fuentes). Puede ser igualmente aplicada en la manera de observar, un punto de vista de quien informa. Para el autor, el ángulo debe ser definido como la manera específica, la selección de las cuestiones y de las formas que un periodista emplea para interrogar la complejidad de lo real. 
la Agencia Senado de Noticias y en el Diario del Senado, por ejemplo, la lectura de la producción interna es rutina obligatoria según el Manual de Redacción) y un segundo nivel, externo, en que los periodistas de los MFSF se alimentan de las informaciones de la prensa tradicional. En Radio Senado la lectura permanente de los diarios y revistas es formalmente recomendada, una forma de calificación permanente para el buen desempeño profesional del radioperiodista.

La retroalimentación se da en diversos momentos del proceso de construcción de la noticia desde el proceso de definición y selección de pautas a ser cubiertas hasta el cierre final del noticiero, sea impreso o audiovisual. En la investigación con los periodistas de las fuentes se constató que $90 \%$ tiene por hábito informarse previamente sobre el asunto leyendo otros diarios de la prensa tradicional. En el trabajo de Producción, citada anteriormente, tienen peso importante los portales de Internet y la lectura de diarios y agencias de noticias.

La retroalimentación interna tiene dimensiones menores. La Agencia Senado de Noticias es la fuente de información más buscada. Ocho de cada diez periodistas tienen el hábito de informarse por ella previamente. ${ }^{19}$ El porcentaje de lectura del contenido del Diario del Senado es de $60 \% .^{20}$ Internet se reveló igualmente como una importante fuente para ese grupo de profesionales. Tanto los portales de instituciones externas (94\%), como el portal del propio SF (70\%) son fuentes rutinarias de obtención de informaciones previas.

Las páginas externas en la web tienen grado de importancia superior al de las fuentes personales consideradas confiables (80\%), y se muestran más importantes que los compañeros de profesión, que son normalmente consultados por seis de cada diez profesionales del Senado. Entre los sectoristas, $88 \%$ tienen el hábito de consultar portales en la web y $58 \%$ se

19 En el grupo de control, $96 \%$ de los periodistas acreditados escuchados tienen el hábito de informarse leyendo otros diarios, 30\% leen el Diario del Senado y $60 \%$ la Agencia Senado.

20 En la obtención de este porcentaje se consideró solamente las respuestas de los profesionales que trabajan en los demás medios del Senado, sin considerar aquellos que trabajan en el Diario del Senado. 
informan rutinariamente en el portal del Senado federal. ${ }^{21}$ Los objetivos de esta lectura serían obtener un mejor conocimiento de la actualidad y favorecer la confrontación de diversas interpretaciones. En la realidad periodística nacional, la consulta a Internet se está consolidando como una práctica normal de los profesionales (Del Bianco, 2004: 6). De esta forma, el periodista cree que está reduciendo su grado de inseguridad sobre un determinado tema y busca no ser superado por la competencia.

Este perfil revela una característica que refuerza la importancia para esos profesionales de trabajar con informaciones seguras, consideradas como oficiales. En contraste, para $96 \%$ de los periodistas sectoristas, la consulta a las fuentes personales confiables es la principal rutina. Por los números obtenidos, tienden a no consultar a sus colegas de profesión, lo que se justifica por la cultura de la información exclusiva, la "exclusiva periodística", de la competencia entre medios y profesionales. ${ }^{22}$ En los MFSF no existe esta preocupación, pues su producción será distribuida a la prensa de manera general, además, claro, de la difusión directa al público.

21 Este portal tiene por costumbre ofrecer la agenda de los trabajos parlamentarios, votaciones en las comisiones y en el pleno, textos de proyectos de ley, base jurídica, currículos de los parlamentarios, etcétera.

22 La existencia de un sistema competitivo entre los sectoristas que cubren el Congreso Nacional no es aceptada unánimemente. El nivel de exigencia y de inseguridad en la prensa tradicional, donde no hay estabilidad en el trabajo, habría propiciado el surgimiento de un mecanismo de autopreservación profesional. Como hay seguridad de que sus textos abarcan los mismos temas del otro periodista con el cual compite, el riesgo de omisión de un tema, de "no dar una noticia", y, en consecuencia, de sufrir sanciones, es menor. Según el periodista Augusto Nunes, que ya pasó por la dirección de periodismo de varios medios brasileños, "está formado en el Congreso Nacional, un verdadero pool de periodistas para evitar que cualquier compañero deje de dar una noticia. Al final de la tarde hay una reunión de periodistas (de medios diferentes) que intercambian frases: por eso uno lee el mismo reportaje sobre el Congreso en todos los diarios" (en Vieira, 1991: 28). Para más detalles sobre el funcionamiento de la prensa en un sistema de hipercompetencia, ver Charron y De Bonville, 2004: 273-316. 
La influencia permanece, en mayor o menor grado, durante el proceso de apuración y redacción de la noticia. La gran mayoría de los profesionales escuchados no ve problemas al realizar su trabajo en informarse a la distancia o seguir los hechos a través de transmisión radiofónica o televisiva de alguna otra emisora tradicional (84\%) y mucho menos si la transmisión radiofónica o televisiva es de la Radio y/o la Tele Senado (94\%).

Existe, sin embargo, entre los periodistas del Senado, una resistencia más grande en aprovechar citas entre comillas no presenciadas por el profesional, pero divulgadas en otro medio de la prensa tradicional. Mientras $74 \%$ del grupo rehusa tal práctica, la resistencia entre los sectoristas cae a $66 \%$. El cuadro se invierte cuando se refiere a algún MFSF. La confianza entre los pares hace que $70 \%$ admitan la posibilidad de usar una declaración, una cita entre comillas, no presenciada personalmente, pero divulgada en algún medio institucional del SF. Lo mismo ocurre con $64 \%$ de los integrantes del grupo de control.

El hecho de que seis de cada diez periodistas sectoristas se valgan de los contenidos de los medios del Senado revela que la prensa tradicional deposita credibilidad en los medios de la fuente en cuestión. Esta conclusión se refuerza por el hecho de que nueve de cada diez de la prensa tradicional admiten la posibilidad de seguir e informarse a distancia sobre los hechos que irán a cubrir a través de transmisión radiofónica o televisiva de la Radio o la Tele Senado. Es una confianza superior a la depositada en las emisoras de radio y televisión tradicionales. Esta segunda situación es admitida por $80 \%$ de los periodistas consultados.

El proceso de retroalimentación se da igualmente después de la redacción de los textos. El hábito de verificar y comparar la producción personal con la de los medios que le hacen competencia alcanza $63 \%$ de los profesionales de los MFSF y se amplía a un universo de $66.7 \%$ cuando la revisión se da en relación con las informaciones producidas por los medios internos. Entre aquellos que comparan sus textos con los de la prensa tradicional, $75 \%$ admite que en la existencia de datos nuevos alterarían o complementarían su propio texto. Este sería el mismo comportamiento adoptado por $80 \%$ de aquellos que hacen la verificación de contenidos en el ámbito de los medios internos del Parlamento.

El hábito es el mismo entre los sectoristas, pues $84 \%$ comparan sus producciones con los medios que les hacen competencia y $38 \%$ con el 
contenido de los MFSF. Para el primer caso, uno de cada dos periodistas que analiza la producción de los otros medios, alteraría o complementaría sus textos y, en el segundo caso, la alteración del texto abarca $74 \%$ de los periodistas que poseen el mismo hábito. No se identificó la razón de esta práctica, pero el temor de no divulgar una información completa, sin errores, puede estar en la raíz de la causa.

\section{INTERLOCUTORES DE LOS PERIODISTAS}

La encuesta identificó que los dos grupos de periodistas viven un confinamiento, un "aislamiento profesional" común a la realidad nacional del sector (Herscovitz, 2000: 78). La principal interlocución de los periodistas del Senado es "intramuros". En una escala de cuatro puntos, los compañeros de trabajo destacan con 3.8 puntos y las jefaturas superiores con 3.7.23 Los periodistas de otras empresas (2.7) y los parientes (2.69) forman el segundo pelotón de interlocutores predilectos. Prácticamente, no existe un feedback por parte de los lectores (2.43) y de las fuentes (2.2). Un escenario semejante fue identificado en el grupo de control. ${ }^{24}$ En este grupo, sin embargo, las fuentes (2.52) son interlocutores más importantes que los parientes (2.48) y el público (2.48).

Las conversaciones intramuros, con jefes y compañeros de trabajo, e intracorporis, con profesionales de otros medios, son las más representativas en ambos grupos. La prevalencia de comunicaciones intramuros en todos los grupos investigados refuerza la idea de la existencia, en la realidad periodística nacional, del fuerte peso de los niveles jerárquicos superiores en el proceso de construcción de la noticia, principalmente, para los profesionales más jóvenes. El profesional nuevo busca adoptar formas comportamentales e incorporar trazos de identidad esenciales para ser aceptado en el medio, así como para construir un futuro profesional (Negreiros, 2004: 100).

Por otra parte, el diálogo intracorporis sugiere una búsqueda de reconocimiento profesional (Sousa 2000:56). El prestigio de los perio-

23 Los cuatro niveles son: 1 = inexistencia total de comunicación, $2=$ raramente; 3 = ocasionalmente y $4=$ frecuentemente.

24 Compañeros de trabajo, 3.6; jefes y superiores, 3.54; periodistas de otras empresas, 2.73 . 
distas no es reconocido por sus títulos académicos, sino por el reconocimiento social de su competencia, principalmente entre los compañeros de profesión (Rieffel, 1984). Es eso lo que les aporta respeto y credibilidad profesionales. La consulta a otros periodistas refleja un nivel de sensibilidad acentuado en la corporación, una necesidad constante de confirmar con los compañeros las percepciones que tienen con relación a los hechos (Sousa, 2000: 56).

El reconocimiento social intracorporis todavía es más necesario en el caso de los periodistas de las fuentes, pues este segmento profesional es el fruto de un proceso históricamente reciente. Si no hay a priori un reconocimiento por parte de la colectividad periodística que esta actividad se inserta en el territorio profesional de los periodistas, difícilmente la sociedad lo verá como tal. ${ }^{25} \mathrm{El}$ reconocimiento por el medio periodístico permite además generar una referencia ante los demás medios de comunicación. Este diálogo corporativo representa una inversión capaz de destacar al profesional e insertarlo en el mundo de las importantes fuentes periodísticas, el llamado establishment-políticos, empresarios, expertos, etcétera. Un profesional con buen concepto consigue abrir más fácilmente las puertas que dan acceso a las informaciones más importantes.

Esta búsqueda de reconocimiento externo envuelve incluso la participación en concursos nacionales e internacionales de periodismo. Radio Senado ya fue galardonada con la medalla de plata del concurso Unca Awards for Excellence in Journalism (2005), promovido por la Organización de Naciones Unidas, en ocasión de sus 60 años de existencia. El mismo año, Tele Senado recibió el Prêmio Margarita de Prata, concedido por la Conferencia Nacional de los Obispos de Brasil a trabajos periodísticos sobre el tema "inclusión social", y varios de sus documentales fueron preseleccionados en otros concursos diversos.

\section{NIVEL DE SATISFACCIÓN}

El modelo productivo de los MFSF no parece afectar negativamente el nivel de satisfacción de los periodistas que actúan en ellos. Es expresivo el volumen de los periodistas que están "muy satisfechos" con la pro-

25 Para más información sobre reconocimiento del territorio profesional consultar Ruellan, 1993: 47. 
fesión, 57\%, y que están "razonablemente satisfechos", 27.45\%. Prácticamente nueve de cada diez periodistas muestran satisfacción con su empleo. Es más que el doble verificado en el grupo de control. No existe una razón preponderante que sea la base de este nivel de satisfacción. Entre los cinco motivos más citados están: el sueldo recibido (23\%), el nivel de autonomía profesional y la libertad de expresión en el trabajo (18\%), y empatados en una tercera posición están tres motivos, cada uno representando $16 \%$ de los profesionales: la seguridad en el empleo, la posibilidad de ayudar a las personas, a la sociedad, a la naturaleza y el tipo de producto periodístico que realiza.

Además de presentar un volumen de satisfacción superior, esos profesionales tienen razones diferentes cuando son comparados con la prensa acreditada. La autonomía profesional, asociada a la capacidad de influenciar asuntos públicos son las razones de satisfacción de prácticamente seis de cada diez periodistas de la prensa tradicional. Cuestiones de carácter material, como nivel del sueldo y seguridad en el empleo, presentan un peso menos significativo, $39 \%$.

\section{CONCLUSIÓN}

Un primer balance nos evidencia que el patrón social de los dos grupos es semejante. Sin embargo, hay diferencias en el nivel de educación universitaria, distribución racial y la forma de ejercer la ideología. Los periodistas de los MFSF se mostraron más vinculados a partidos y al sindicato del sector, que es el mismo para los dos segmentos. Este grupo de periodistas es sometido a un modelo de producción de la noticia con libertad limitada. Un modelo que no permite la expresión de puntos de vista o análisis personales. Su trabajo es fuertemente jerarquizado, sujeto a las normas regidas por aquella Casa del Parlamento y a la jerarquía interna de las redacciones. La producción de contenidos sigue una lógica periodística, un patrón de noticia con poco espacio para la participación en el proceso deliberativo de los llamados profesionales de punta-reporteros, redactores, productores, etcétera.

En términos de estilo periodístico, el discurso consiste, preferencialmente, en un relato seco y objetivo de los hechos, sin análisis de carácter personal, pero respetando la diversidad ideológica y partidaria existente en el Parlamento. El hecho de ejecutar un periodismo descrip- 
tivo no excluye este género informativo de la categoría periodismo. El grado de crítica de un texto no es el elemento que define la naturaleza periodística de una información. El contenido del discurso es variable por naturaleza, pero los códigos que regulan la acción son estables por definición (De Bonville, 2004: 294). 26

Esas características no difieren radicalmente de las normalmente encontradas en el campo periodístico. El periodista será siempre presionado por la política editorial y por la forma de hacer las cosas en el órgano de comunicación para el cual trabaja (Sousa, 2000: 58). El savoir-faire de todos los periodistas se construye y se manifiesta en un espacio de presiones y restricciones y en una estructura de interdependencia, con los niveles jerárquicos más elevados, colegas y fuentes (Néveu, 2004: 43). La "noticia" resulta, así, de un proceso organizado y presionado por fuerzas internas y externas. De esta forma, el modelo de producción periodística de los medios de las fuentes del Senado federal no difiere en género de la regla universalmente utilizada por los medios de comunicación, pero tal vez lo haga en cuestión de grado.

El periodismo practicado por los medios legislativos puede ser considerado "legalista", por no sumar reflexión u opinión personal a las emitidas por las propias fuentes (Lemieux, 1992: 31-33). Por su parte, Jardim (2006: 16) prefiere clasificarlo como portador de un perfil "informativo y equlibrado". Una eventual sospecha sobre su contenido no puede basarse solamente en el hecho de que los periodistas trabajen para sus fuentes. En el periodismo, de manera general, la relación "fuente-periodista" no es obligatoriamente de oposición, sino puede ser de "identidad o complementariedad" (De Bonville, 2001: 30), y resultar en la generación de intereses y de satisfacciones comunes a los dos polos de acción informativa (Gans, 1979: 116).

Los periodistas no requieren concentrarse obligatoriamente sólo en los aspectos negativos del poder (Sousa, 2000: 110) y tampoco estos medios dejan de informar los casos escabrosos, como corrupción y desmanes

26 Aquí nos valemos de la noción de código explicitada por De Bonville, no solamente como un sistema de reglas que reglamentan el discurso periodístico (estilo, género, jerarquización de la información, etcétera), sino también como una reglamentación de práctica y comportamiento profesional (Charron y De Bonville, 2004: 282). 
políticos. La misión profesional de los llamados "periodistas neutrales" se limita a recoger, procesar y difundir lo más rápido posible las informaciones. O sea, sería un periodismo donde el profesional asume una condición de canal de transmisión. Una tarea complementaria a la de "perro de guardia", por la cual el profesional es más "participativo", investigador y explorador (Johnstone, Slawski y Bowman, 1972: 522-540). La misión de "perro de guardia" en estos medios legislativos es traspasada al propio público, que la ejerce a través de las transmisiones en vivo, sin cortes o edición, de lo que ocurre en el Parlamento (Jardim, 2006: 8). Los medios de comunicación de masa de los parlamentos constituyen, así, un instrumento de la sociedad al proporcionar nuevas bases de información y de seguimiento de las actividades legislativas (Renault, 2004: 130).

Las barreras internas del Senado al periodismo de opinión tienden a ser sobrepasadas, o por lo menos compensadas, por una inserción más plural de fuentes, y por medio de una fuerte presencia de programas de debates, de entrevistas, de programas especiales temáticos y de documentales. Programas normalmente ausentes de los medios privados nacionales, cuyo modelo comercial de programación impone espacios más pequeños a productos mediáticos de este perfil.

El periodismo practicado por los MFSF contribuye a un proceso de transparencia, a una acción de accountability y es complementario del modelo de investigación de la prensa tradicional. La encuesta reveló que aunque no tenga esta naturaleza de investigación, los profesionales de la prensa tradicional le dan credibilidad al contenido informativo difundido por los MFSF. La realidad reciente de Brasil también demuestra que en algunos casos, no raros, fue la propia prensa tradicional la que ejecutó un periodismo burocrático o incluso el mismo emisor omitió estratégicamente temas que no consideraba oportunos. En otros casos, son los medios de las fuentes los que han revelado temas e informaciones socialmente importantes, despreciados por los medios tradicionales. 27

27 En el 2004, prácticamente toda la prensa nacional ignoró los trabajos de la Comisión de Investigación Parlamentaria del Banestado, que estudiaba el envío ilegal de dólares al extranjero. Las denuncias llegaban demasiado cerca de los principales medios de comunicación. Solamente los MFSF y la Red Record de Televisión cubrieron el hecho. 


\section{Bibliografía}

ADGHIRNI, Z. (2004). Valores-notícia e credibilidade no jornalismo online, comunicación científica hecha en ocasión del Congreso de la Sociedad Brasileña de Investigadores en Periodismo. Brasilia: SBPJOR.

BOURDIEU, P. (1997). Sobre a televisão. Rio de Janeiro: Jorge Zahar.

- (1980). Le sens pratique. Paris: Éditions de Minuit.

Charron, J. (2002). Parler de soi en faisant parler les autres. Identité journalistique et discours rapporté. En Rieffel, R. et Watine, T. Les mutations du journalisme en France et au Québec (pp. 83-117). Paris: Panthéon Assas.

— et De Bonville, J. (2004). Le journaliste et le marché: de la concurrence à l'hyperconcurrence. En Brin, C., Charron, J. et De Bonville, J. (dir.), Nature et transformation du journalisme - théorie et recherches empiriques (pp. 273-316). Laval: PUL.

COMSEFE. (2003). Estatuto Social da Associação dos Profissionais de Comunicação do Senado Federal. Brasília. Disponible in: http:// f1.grp.yahoofs.com/v1/sLhRRfnh4HigGBvbwB9-WJt_Cd22jJIZHMiKlLFDW7E1G769XfBqs 1 ehrVxDQXdWNB7zShv6D8_eqfZi O9OAQ/Estatuto\%20da\%20Comsefe.htm

DE BONVILLE, J. (2001). Le journalisme dans le "systeme" médiatique. Laval: mimeo.

- (2004). Le métier de journaliste au début du 20e siècle vu par un contemporain: de l'utilité des concepts théoriques. En Brin, C., Charron, J. et De Bonville, J. (dir.). Nature et transformation du journalisme - théorie et recherches empiriques (pp. 243-272). Laval: PUL.

DEL BIANCO, N. (2004). Noticiabilidade no rádio em tempos de Internet. En Anais do VI Lusocom. Corvilhão: Lusocom.

FREITAS, L. C. (2004). A midiatização do parlamento (disertación de maestría en comunicación social). Brasília: Faculdade de Comunicação da Universidade Brasília.

GANS, H. (1979). Deciding what's news. New York: Vintage Books.

HeRSCOVITZ, H (2000). Jornalistas de São Paulo: quem são e o que pensam em comparação aos jornalistas americanos e franceses. Revista Brasileira de Ciências da Comunicação, vol. XXIII, num. 2, jul/dic, (pp.65-86). São Paulo: Intercom. 
JARDIM, M. de A. (2006). O mapa da mídia eletrônica legislativa na América Latina (Comunicación científica realizada en ocasión del 3er Congreso Latinoamericano de Ciencia Política). Campinas: Brasil, sept.

Johnstone, J., Slawski, E. y Bowman, W. (1972). The professional values of American newsmen. Public Opinion Quarterly, vol. 36, núm. 4, pp. 522-540.

LEMIEUX, C. (1992). Les journalistes, une morale d'exception? En Bourmeau, S., Cardon, D. et Le Gavre, J. (dir.), L'activité journalistique. Paris: Presses Fond. Nat. des Sciences Politiques.

MATHIEN, M. (1992). Les journalistes et le système médiatique. Paris: Hachette.

MCMANE, A. (1992). Vers un profil du journaliste occidental". Réseau, num. 51, (pp. 67-73). Paris: CENT.

NEGREIROS, J. (2004). Fantasmas ao Espelho - modo de auto-representação dos jornalistas, Coimbra: Minerva Coimbra.

NÉVEU, É. (2004). Sociologie du journalisme. Paris: La Decouverte.

PASQUALI, L. (1996). Teoría e métodos de medida em ciências do comportamento. Brasilia: Universidade de Brasilia/Instituto Nacional de Estudos e Pesquisas Educacionais.

RÁDIO Senado, Manual de redação (2000) Consultado el 13 de octubre de 2005, http://www.senado.gov.br/radio.

RENAUlT, L. (2004). Comunicação e política nos canais de televisão do Poder Legislativo no Brasil. Belo Horizonte: ALMG.

RIBEIRO, J. C. (1994). Sempre Alerta. S.Paulo: Brasiliense.

RIEFFEL, R. (1984). L'élite des journalistes. Paris: PUF.

- (2001). Vers un journalisme mobile et polyvalent? Quaderni num. 44, automne, pp. 153-169.

RUELLAN, D. (1993). Le professionnalisme du flou - identité et savoirfaire des journalistes français. Grenoble: PU de Grenoble.

- (2001). Socialisation des journalistes entrant dans la profession. Quaderni, núm. 44, automne, pp. 137-152.

SANT'ANNA, F. (2005). Midia das fontes - o difusor do jornalismo corporativo. Brasília: Casa das Musas.

SCHLESINGER, P. (1983). Os jornalistas e a sua máquina do tempo. En Traquina, N. (org.), Jornalismo: questões, teorias e "estórias". Lisboa: Veja. 
- (1987). Putting 'Reality' Together, BBC news. London: Routledge. SCHUDSON, M. (1988). Por que é que as notícias são como são. Jornalismos - Comunicação e Linguagens, num. 8, pp. 17-27, Lisboa.

SENAdO FEDERAL, (2002). Ato da Comissão Diretora, num. 15, 2002. Boletim administrativo do pessoal, núm. 2541, Brasilia, 16 de mayo.

- (2003). Manual de Redação - Agência Senado e Jornal do Senado, Brasilia: Senado Federal.

SousA, J. P. (2000). As notícias e seus efeitos. Coimbra: Minerva Coimbra.

THOMPSON, J. B. (2005). La nouvelle visibilité. Visibilité/Invisibilité, Réseaux, vol. 23, num. 129-130, pp 59-87. Paris: FT R\&D/Lavoisier.

TraVAnCAS, I. (1983). O mundo dos jornalistas. São Paulo: Summus.

TUCHMAN, G. (1972). Objectivity as strategic ritual: an examination of newsmen's notion of objectivity. American Journal of Sociology, vol. 77, núm. 4, New York.

TV SENADO (1999). Manual de redação. Consultado el 13 de octubre de 2005, http://www.senado.gov.br/tv/conheca/manual/intro.htm

VIEIRA, G. (1991). Complexo de Clark Kent - São super-homens os jornalistas?. São Paulo: Summus.

WEAVER, D. H., \& Wilhoit, G. C. (1986). The American journalist: A portrait of U.S. news people and their work. Indiana: University Press, Bloomington .

— \& Wilhoit, G. C. (1996). The American journalist in the 1990s, U.S. news people at the end of an era. Mahwah: Lawrence Erlbaum. 